\title{
First-line treatment of acute lymphoblastic leukemia with pegasparaginase
}

\author{
This article was published in the following Dove Press journal: \\ Biologics:Targets \& Therapy \\ 7 July 2009 \\ Number of times this article has been viewed
}

\section{Riccardo Masetti \\ Andrea Pession \\ Pediatric Oncology and Hematology Unit "Lalla Seràgnoli", University of \\ Bologna, Bologna, Italy}

\begin{abstract}
Acute lymphoblastic leukemia (ALL) accounts for almost 4000 cases annually in the United States, approximately two thirds of which are in children and adolescents. Treatment results of ALL have improved considerably in the past decade, due to an optimal stratification of patients and a rational use of different antileukemic agents among which L-asparaginase (L-ASNase) plays a fundamental role. This drug has been used in pediatric ALL chemotherapy protocols for almost 3 decades. In the 1970s and 1980s a chemically modified form of this enzyme called pegasparaginase (PEG-ASNase) was rationally synthesized to decrease immunogenicity of the enzyme and prolong its half-life. The different advantages of PEG-ASNase have been demonstrated in many clinical studies, the last of which underline the utility of this drug in front-line therapy of ALL. In this review, we discuss the pharmacological advantages and clinical potential of PEG-ASNase and its important use in first-line treatment
\end{abstract} of ALL.

Keywords: pegasparaginase, acute, lymphoblastic leukemia, pegylation

\section{Introduction}

Acute lymphoblastic leukemia (ALL) is a heterogeneous group of disorders that result from the clonal proliferation and expansion of malignant lymphoid cells in the bone marrow, blood and other organs. ${ }^{1}$ This is the most common type of cancer in children and adolescents accounting for $23 \%$ to $25 \%$ of all malignant diseases. ${ }^{2}$ Pui et al report that in the 1990s, the 5-year event-free survival (EFS) rates for childhood ALL generally ranged from $70 \%$ to $83 \%$ in developed countries with an overall cure rate of approximately $80 \% .^{2}$ Unfortunately, the experience with adult ALL has been far less rewarding but treatment results have improved considerably in the past decade with an increase of complete remission (CR) rates to $85 \%$ to $90 \%$ and overall survival (OS) rates to $40 \%$ to $50 \% .{ }^{3}$ For all patients, specific treatment approaches differ but generally consist of remission-induction therapy followed by intensification (or consolidation) therapy and continuation treatment. Central nervous system (CNS)-directed therapy, given for varying lengths of time depending on the patient's risk, is administered in order to prevent meningeal progression or relapse.

L-asparaginase (L-ASNase) is an important antileukemic agent used in first-line treatment of a variety of lymphoproliferative disorders. It has been used in treatment of pediatric ALL for almost 30 years. ${ }^{4-8}$ L-ASNase was identified as a potential antileukemic agent in 1961 when it was isolated as an antilymphoma component of guinea pig serum..$^{9,10}$
Correspondence: Andrea Pession Oncologia ed Ematologia Pediatrica "Lalla Seràgnoli”, Clinica Pediatrica, Università di Bologna, Via Massarenti, I I, 40I38, Bologna, Italy Email andrea.pession@unibo.it 
In 1964 the asparaginase of bacterial origin was isolated. Mashburn reported the purification of Escherichia coli L-asparaginase (EC-L-ASNase) and demonstrated its tumoricidal activity. ${ }^{11}$ Currently, 3 asparaginase formulations are available in the United States: E. coli native L-ASNase, erwinase and pegasparaginase (PEG-ASNase). The antitumor effect of this enzyme results from the depletion of asparagine (ASN), an amino acid essential to lymphoblast leukemic cells leading to an inhibition of protein synthesis with consequent considerable cytotoxicity.

It has been also demonstrated that application of extended high dose of L-ASNase may compensate reduced leukemia control resulting from adoption of a reduced intensity chemotherapy schedule (Berlin-Frankfurt-Munster backbone) for treatment of children with standard risk ALL. ${ }^{12}$ The role of L-ASNase as an antileukemia drug has been a matter of discussion due to the high rate of allergic reactions. The major limitation to the use of L-ASNase is dose limiting clinical hypersensitivity, which occurs in $3 \%$ to $78 \%$ of patients treated with unmodified forms of enzyme. ${ }^{6-15}$ However, these clinical complications seem to have been solved by use of modified versions of L-ASNase: polyethylene glycol (PEG)-conjugated asparaginase (pegasparaginase) has significant pharmacological advantages over native EC-L-ASNase and also allows adequate plasma enzymatic activity and asparagine depletion, ${ }^{16}$ and can be substituted in cases of hypersensitivity to native L-ASNase. Coupling of the enzyme to PEG was identified as a process by which the immunogenic reactions of the drug were diminished without altering its antineoplastic property. ${ }^{17,18}$ PEGylation also increases drug stability and the retention time of the conjugates in blood, and reduces proteolysis and renal excretion, thereby allowing a reduced dosing frequency. ${ }^{19}$ Thus, conjugating the native EC-L-ASNase molecule to PEG has provided multiple advantages such as: a definite reduction of immunogenic properties, increased nonreactivity to antibodies, a considerably longer half-life and a reduction of the number of injections for the patient. In recent years, clinical trials have established the importance of PEG-ASNase in frontline pediatric and adult ALL therapy. In this review we discuss about the advantages of PEG-ASNase and its use in first-line treatment of ALL.

\section{Chemistry and pharmacology of the drug \\ Chemistry of the drug and pegylation}

L-ASNase has been produced in large quantities from two bacterial species, E. coli and Erwinia caratovora. The enzyme obtained from these two sources has been found to have lowest toxicity among various similar enzymes. ${ }^{20}$ E. coli produces two asparaginases, type EC-1 and EC-2. ${ }^{21}$ The purified $E$. coli enzyme has a molecular weight of 133 to $141 \mathrm{kDa} .^{22,23}$ The chemical structure of all asparaginases is composed of four identical subunits, A, B, C, and D with an active site on each subunit. The molecular weight of each subunit is $22 \mathrm{kDa} .{ }^{24}$ Subunits A and B and subunits C and D form extensive interactions, resulting in dimers. Each dimer has two active sites which contain an aspartate residue, but only the tetrameric form has enzymatic activity. ${ }^{25,26}$ The specific activity of purified enzyme is between 300 and $400 \mu \mathrm{mol}$ of substrate per minute per milligram of protein.

The process known as pegylation, which consists of conjugating biomolecules with PEG, is a well-established technology for increasing the circulating half-life of protein and liposomal pharmaceuticals. In general the advantages of PEGylation technology are increased bioavailability, increased blood circulation of the drug, optimized pharmacokinetics (see below), decreased immunogenicity and decreased frequency of administration. ${ }^{19}$ PEGs are non-toxic, water-soluble polymers that create a shield around the pegylated drug, preserving it from renal clearance, enzymatic degradation, and recognition by cells of the immune system. ${ }^{27}$ Pegylation of L-ASNase was developed in the 1970s and 1980s. Abuchowski et al ${ }^{17}$ were first to successfully couple PEG to L-ASNase. PEG-ASNase is formed by the covalent conjugation of EC L-ASNase (L-asparagine amidohydrolase, type EC-2, EC 3.5.1.1) to monomethoxypolyethyleneglycol (total PEG portion molecular weight $5 \mathrm{kDa}){ }^{28-30}$ PEG-ASNase, which is formed by 4 identical subunits with interactions resulting in dimmers like EC L-ASNase, has similar chemical properties to EC L-ASNase with optimal conditions for activity at $\mathrm{pH} 7.0$, and reaction temperature of $50{ }^{\circ} \mathrm{C}$ isoelectric point at $\mathrm{pH} 5.0{ }^{30}$ Pegylation covers immunogenic epitopes, reducing hypersensitivity reactions associated with EC L-ASNase and allowing free access of ASN to the active sites of the enzyme while limiting EC L-ASNase's uptake by the reticuloendothelial system, protecting its antigenic determinants from immune detection. ${ }^{27,31}$ This also delays the elimination of the enzyme by means of reticuloendothelial system, prolonging the half life of the drug. ${ }^{27,29,32}$ In the US, PEG-ASNase is manufactured by Enzon from EC L-ASNase obtained from Merck and Co. Inc. In Europe the PEG-ASNase product is derived from the Kyowa Hakko native asparaginase protein. ${ }^{33-36}$ Commercially available preparations for therapy are known generically as pegaspargase; the trade name is Oncaspar ${ }^{\circledR}$ (Enzon, South Plainfield, NJ, USA). 


\section{Antineoplastic action}

The antineoplastic action of L-ASNase is based on the assumption that tumor cells, especially lymphatic cells, require a huge amount of ASN to maintain their rapid malignant growth. L-ASNase catalyzes the hydrolysis of L-ASN to L-aspartic acid and ammonia. . $^{37,38}$

L-ASNase has a significant effect in depletion of serum ASN and kills tumor cells by depriving them of an essential factor required for protein synthesis. Both EC L-ASNase and PEG-ASNase hydrolyze asparagine into aspartic acid and ammonia and deplete circulating asparagines from plasma, the main exogenous sources of ASN.$^{39-41}$ Leukemic cells lack sufficient asparagine synthetase compared with normal cells, so they cannot re-synthesize the asparagine de novo. ${ }^{42}$ Cell cycle arrest in the $\mathrm{G} 1$ phase has been documented in the murine L5178Y cell line ${ }^{43}$ and also in the MOLT-4 human T-lymphoblastoid line, resulting in apoptosis. ${ }^{44}$

\section{Pharmacokinetics and pharmacodynamics}

In vivo studies revealed that the half-lives of Erwinia and EC L-ASNase are similar, with 10 hours as mean half-life..$^{45}$ The drug seems to remain confined to the vascular space after administration. It has been detected in the pleural fluids and ascites ${ }^{46}$ but not in cerebrospinal fluid (CSF). ${ }^{47}$ PEG-ASNase, as well as native L-ASNase, has pharmacokinetic properties that include a monophasic half-life, a one-compartment model and a single elimination phase. ${ }^{48}$ The peak and trough levels and the area under the curve of PEG-ASNase are proportional to the dose. The distribution volume, clearance and half-life of PEG-ASNase are not dose dependent. ${ }^{49-51}$ The elimination half-life of PEG-ASNase is approximately 5.5 to 6 days, 5 times longer than that of EC L-ASNase and 9 times longer than that of Erwinia ASNase.

Comparative half-lives of different types of L-ASNase are listed in Table 1. ${ }^{49,50,52}$ PEGylation, as shown in Table 1, confers a markedly prolonged half-life to the drug. Table 1 (last row) shows 5 patients who presented hypersensitivity reaction to EC L-ASNase with a decreased serum $T_{1 / 2}$ (hours) of PEG-ASNase given at $2500 \mathrm{IU} / \mathrm{mq}$. This confirms that the pharmacokinetics of PEG-ASNase may be adversely affected by the development of antibodies directed against the ASNase portion. Modified enzyme is cleared more rapidly in patients with hypersensitivity, although the $T_{1 / 2}$ remains longer than that of the native enzyme..$^{50}$ Armstrong et al studied the effects of the presence of anti-PEG antibodies that are discussed below. ${ }^{63}$ The presence of anti-PEG antibodies was highly associated with rapid clearance of PEG-ASNase.
Table I Pharmacokinetic studies of patients undergoing therapy with L-asparaginase

\begin{tabular}{lllll}
\hline $\begin{array}{l}\text { L-asparaginase } \\
\text { preparation }\end{array}$ & $\begin{array}{l}\text { No. } \\
\text { patients }\end{array}$ & $\mathbf{T}_{1 / 2}(\mathbf{h})$ & $\begin{array}{l}\text { Dose range } \\
\text { (IU/mq) }\end{array}$ & Ref. \\
\hline E. coli & 9 & $34 \pm 8$ & $2500-25000$ & 50 \\
E. coli & 7 & $20 \pm 6$ & - & 49 \\
E. coli $^{\mathrm{a}}$ & 5 & Undetectable & 25000 & 50 \\
Erwinia $^{\mathrm{b}}$ & 10 & $16 \pm 3$ & 25000 & 50 \\
Erwinia $_{\text {PEG }}$ & 13 & $6,4 \pm 5$ & 30000 & 52 \\
PEG & 25 & $357 \pm 243$ & $500-8000$ & 49 \\
PEG & 10 & $138 \pm 78$ & 2500 & 50 \\
\hline
\end{tabular}

aPatients with previous hypersensitivity to $E$. coli L-asparaginase. bIntravenous administration (all others intramuscular).

'Patients with previous hypersensitivity to E. coli L-asparaginase, treated with PEG-ASNase.

On the route of administration of PEG-ASNase, the adult and pediatric clinical trials reported ${ }^{16,53,54,93,97}$ show that intravenous (iv) administration can be used, without higher incidence or severity of allergic or nonallergic side effects than reported for intramuscular (im) or subcutaneous administration. The iv route of administration will eliminate painful injections and achieve rapid peak levels and asparagine depletion. Following the im administration of PEG-ASNase $\left(2500 \mathrm{UI} / \mathrm{m}^{2}\right)$ the serum levels of ASN fall by 4 days and remained depleted for around 3 weeks. ${ }^{48}$ After iv administration PEG-ASNase deamination of ASN occurred by 2 hours after administration and was sustained for $\sim 3$ weeks (dose of $2000 \mathrm{UI} / \mathrm{m}^{2}$ ) in adult ALL patients ${ }^{53}$ and for $\sim 5$ weeks (dose of $2500 \mathrm{UI} / \mathrm{m}^{2}$ ) in pediatric ALL patients. $^{54}$

PEG-ASNase penetrates poorly into the CSF. ${ }^{16,25,48}$ However, ASN depletion in the CSF has been documented following both EC L-ASNase and PEG-ASNase administration and may be dose-dependent. ${ }^{25,48,55}$ The CSF ASN level is known to rebound once L-ASNase therapy is completed. $^{16,48}$

\section{Drug resistance}

For L-ASNase and PEG-ASNase, different mechanisms of drug resistance have been recognized. A possible mechanism of resistance to L-ASNase is the development of antibodies that neutralize the enzyme. ${ }^{55,67}$ The development of antibodies against PEG-ASNase and EC L-ASNase can reduce their activity, leading to failure of asparagine depletion after readministration of the drug. ${ }^{50,53,56-58,63}$ PEG-ASNase has been reported to cause the development of anti L-ASNase antibodies in L-ASNase-naive patients treated with PEG-ASP, at a rate 
of up to $12 \%$ in children ${ }^{48,60}$ and $4 \%$ to $15 \%$ in adults..$^{53,62}$ The presence of circulating anti-PEG was very closely associated with rapid clearance of PEG-ASNase, which could consequentially render the treatment ineffective. ${ }^{63}$ The IgG antibody produced can be associated with type II immune allergic reactions. Antibody development may also occur in patients who do not show clinical allergy symptoms (silent hypersensitivity). ${ }^{55,56}$ It has been reported that prior exposure to EC L-ASNase increases the development of anti-PEG-ASNase antibodies, and patients who had developed anti-L-ASNase antibodies to L-ASNase also developed anti-PEG-ASNase antibodies in more than $65 \%$ of cases,${ }^{64}$ but this could be determined by the potential cross reactivity of the IgG antibodies. While the development of antibodies does seem to be correlated with diminished drug effect, the clinical significance of such antibodies is still being debated. ${ }^{56,58,63}$

An increase in ASN synthase activity (the enzyme responsible for the synthesis of l-asparagine in normal cells) has been noted in blasts of patients with ALL clinically resistant to the drug $^{64}$ and in murine lymphoma cells. ${ }^{66,67}$ A concomitant increase in messenger RNA as well as enzyme levels for ASN synthase have been described in several cell lines. ${ }^{68,69}$

Another possible mechanism suspected by Gallagher ${ }^{70}$ is that the pool of L-ASNase sensitive cells may produce cytokines that control the expansion of resistant cells and when sensitive cells are killed by L-ASNase, resistant cells escape from regulatory control.

In 2003 Holleman et al in $2003^{71}$ associated the resistance to different classes of drugs like L-ASNase and prednisolone with impaired apoptosis in childhood ALL: caspase- 3 or PARP [poly(ADP-ribose) polymerase] inactivation seem to be responsible for resistance to these drugs. Moreover, loss of spontaneous caspase- 3 activation in vivo is associated with relapse in adults with ALL. ${ }^{72}$

\section{Toxicity}

The toxicity profile of L-ASNase and PEG-ASNase falls under two main categories, those pertaining to immunological sensitization to a foreign protein, and the adverse events related to the inhibition of protein synthesis. L-ASNase causes little bone marrow depression and usually does not affect the gastrointestinal or oral mucosa or hair follicles.

Toxic hypersensitivity reactions include cutaneous rashes, serum sickness, bronchospasm, and anaphylaxis. ${ }^{73}$ The frequency of these reactions for EC L-ASNase ranges from $13 \%$ to approximately $30 \%{ }^{74}$ Data on file from the manufacturer (Rhone-Poulenc Rorer) of PEG-ASNase report in 174 patients an allergic reaction rate of $10 \%$ in previously nonhypersensitive patients and of $32 \%$ in patients hypersensitive to EC L-ASNase. ${ }^{75}$ Desensitization protocols have been described but have not been studied in large numbers of patients. ${ }^{76}$ The majority of patients experience evidence of hepatotoxicity with an elevation in transaminase and bilirubin levels and abnormal alkaline phosphatase levels have also been reported. ${ }^{57,65} \mathrm{~A}$ decrease in serum albumin, fibrinogen and serum lipoprotein levels is also a manifestation of liver dysfunction. Pathologically, fatty infiltration of the liver has been noted. ${ }^{76}$ Coagulation abnormalities such as low levels of clotting factors have been consistently demonstrated as well as deficiencies of antithrombin III, protein C and protein S. ${ }^{57,77-80}$ Clinically significant hemorrhage due to L-ASNase therapy is very uncommon, while thrombosis of peripheral, pulmonary or central nervous system occurs in up to $10 \%$ of patients. ${ }^{81}$ Congenital procoagulant abnormalities may contribute to the development of thrombic complications; however, the role of central venous catheters and steroid therapy, commonly used in leukemia patients, remains to be clarified. ${ }^{81-83}$ Pancreatitis, characterized by abdominal and/or back pain, anorexia, nausea and vomiting, is a well-documented complication of L-ASNase that occurs in $10 \%$ to $16 \%$ of patients with a mortality rate between $1.8 \%$ and $4.6 \%{ }^{84,85}$ The mechanism of L-ASNase-induced acute pancreatitis is not completely understood. Pathologically, the pancreas is hemorrhagic, suggesting an involvement of coagulation abnormalities. Neurotoxicity, which can occur with symptoms such as depression, lethargy, fatigue, somnolence, confusion, irritability and agitation, is documented in up to $25 \%$ of adult patients treated with 1-asparaginase, ${ }^{86}$ but rarely occur in children. This effect has been suggested to be a result of lack of L-ASN and 1-glutamine in the brain.

A lower incidence of complications was found to be associated with PEG L-ASNase. ${ }^{13,33}$ PEG-ASNase is generally well tolerated by the majority of the patients and grade 3 or 4 toxicity are rare. The primary toxicity of PEG-ASP is to that of immunologic reactions due to linked the exposure to the bacterial proteins. These reactions range from transient flushing or rush and urticaria (grade 1 and 2) to bronchospasm angioedema and anaphylaxis (grade 3-4). ${ }^{33}$ Aside from hypersensitivity, the major side effects of PEG-ASNase are due to inhibition of normal protein synthesis, similar to those of native L-ASNase. 


\section{Clinical trials with PEG-ASNase}

Because of low hypersensitivity and other pharmacological advantages, PEG-ASNase has been the subject of many clinical application in the last 20 years. The PEGylated enzyme has been found to be safe for most patients allergic to EC L-ASNase and the delayed plasma clearance seems to have solved the problem of the need for frequent medication, but some questions need to be readdressed. In this section we report phase I and II clinical trials of PEG-ASNase followed by a discussion of first-line therapy.

\section{Phase I trials}

Ho et al conducted a phase I dose-escalation study in which 31 adult patients received iv PEG-ASNase (dose range 500 to $8000 \mathrm{IU} / \mathrm{m}^{2}$ ) over 1 hour fortnightly. ${ }^{31,49}$ The mean half-life of the drug was 357 hours, suggesting the use of a 2-weekly treatment interval. Three patients developed anaphylactic reactions (one patient from a 500 dose, one from a $2000 \mathrm{IU} / \mathrm{m}^{2}$, one from $4000 \mathrm{IU} / \mathrm{m}^{2}$ ) and hyperglycemia and hepatic dysfunction were other major associated toxicities. This study has been considered the base for subsequent trials, which adopted the same range of doses in between 2000 and $2500 \mathrm{IIU} / \mathrm{m}^{2}$ for clinical studies.

In another phase I study Vieira Pinheiro et $\mathrm{a}^{87}$ infused $500 \mathrm{IU} / \mathrm{m}^{2}$ of PEG-ASNase in children with relapsed ALL, reaching the goal to maintain serum L-ASNase activity (>100 IU/L) adequate for requisite depletion of L-ASN. Most of the patients responded well to this low dose for at least 1 week, suggesting that with careful drug monitoring, lower doses may be administered successfully. Taylor et al in $2001^{88}$ conducted a phase I clinical trial and pharmacodynamic evaluation of PEG-ASNase in patients who had advanced stage solid tumors. They administered PEGASNase at doses of 250, 500, 1000, 1500 and $2000 \mathrm{IU} / \mathrm{m}^{2}$ twice weekly, and obtained in most cases a low level of L-ASN for 14 days. L-ASN levels were extremely low for 2 weeks (except in patients receiving $250 \mathrm{IU} / \mathrm{m}^{2}$ ), with most prolonged depletion at $2000 \mathrm{IU} / \mathrm{m}^{2}$. Grade 1-2 hypersensitivity reactions were most frequently reported at the $2000 \mathrm{IU} / \mathrm{m}^{2}$ dose.

\section{Phase II trials}

In 1995 Ettinger et al reported the results of a multicenter, phase II, open label clinical trial (ASP-201A) conducted with patients with recurrent ALL. ${ }^{33}$ Twenty-one patients received a single dose of pegaspargase $\left(2000 \mathrm{IU} / \mathrm{m}^{2}\right.$ every weeks) during an initial 14-day investigational window then followed by vincristine $1.5 \mathrm{mg} / \mathrm{m}^{2}$ weekly $\times 3$, prednisone
$40 \mathrm{mg} / \mathrm{m}^{2} /$ day $\times 21$, doxorubicin $40 \mathrm{mg} / \mathrm{m}^{2}$ and intrathecal chemotherapy beginning on day 14 . All had previously received L-ASNase. Of the 18 evaluated for response on day 14 after the window with PEG-ASNase monotherapy, $3(17 \%)$ achieved a complete response and $1(6 \%)$ a partial response for a combined response rate of $23 \%$. By completion of the 35-day induction period, 78\% (14 of 18) of evaluated patients achieved complete or partial remission. Five patients experienced mild urticaria and mild local allergic reactions. There was no evidence of anaphylactic problems during the treatment. The incidence of hyperglycemia and pancreatitis was less than expected from the studies with native L-ASNase.

Aguayo et al achieved a completed response rate of $22 \%$ in 22 adults patients with recurrent ALL treated with PEG-ASNase, methotrexate, vincristine and prednisone. The chemotherapy protocol consisted PEG-ASNase $2500 \mathrm{IU} / \mathrm{m}^{2}$ on days 1 and $14 .{ }^{89}$

Douer et al treated 14 adult patients with ALL subjected to $2000 \mathrm{IU} / \mathrm{m}^{2}$ on day 16 of the multi-agent protocol and obtained $93 \%$ complete response. The other agents included vincristine, prednisone and daunorubicine. ${ }^{90}$

Muss et al treated 21 patients with non-Hodgkin's lymphoma with $2000 \mathrm{mg} / \mathrm{m}^{2}$ PEG-ASNase and the drug displayed modest activity in this heterogenous group of patients because only two partial responses were achieved. ${ }^{91}$

\section{First-line treatment of ALL with PEG-ASNase}

Here we review the experience with PEG-ASNase as a front-line treatment for patients with ALL. Randomized clinical trials in children ${ }^{48,92}$ and nonrandomized trials in adults, ${ }^{53,62,90,93}$ demonstrated that substitution of PEG-ASNase for EC L-ASNase in polychemotherapy regimens for ALL has similar efficacy. The Children's Cancer Group (CCG) carried out a randomized comparison between PEG-ASNase $\left(2500 \mathrm{IU} / \mathrm{m}^{2} \times\right.$ single dose on day 1$)$ and native L-ASNase $\left(6000 \mathrm{IU} / \mathrm{m}^{2}\right.$ weekly $\times 3$ doses $) .{ }^{94}$ The overall complete response rates after 4 weeks were comparable: $98 \%$ for PEG-ASNase vs $100 \%$ for the native drug, but PEG-ASNase produced a faster rate of blast clearance than the native drug ( $63 \%$ vs $47 \%$ at day $7,96 \%$ vs $83 \%$ at day 14$)$. Another randomized trial by $\mathrm{CCG}^{48}$ has been conducted comparing EC L-ASNase and PEG-ASNase in treatment of 118 children with newly diagnosed standard-risk ALL. PEG-ASNase was administered at a dose of $2500 \mathrm{IU} / \mathrm{m}^{2}$, im on day 3 of the 4 -week induction phase and on day 3 of 
each of two 8-week delayed intensification phases. Native L-ASNase was administered at a dose of $6000 \mathrm{IU} / \mathrm{m}^{2} \mathrm{im}$ 3 times weekly for 9 doses during induction and for 6 doses during each delayed intensification phase. In patients who received PEG-ASNase, the number of days of asparaginase activity exceeded $>0.03 \mathrm{IU} / \mathrm{mL}$ and was greater than the number of days in native enzyme-treated subjects during both the induction and delayed intensification phases of treatment. With a median follow-up of 3.2 years, the 3-year EFS rates were approximately $80 \%$ in both arms, but even if adverse events, infections, and hospitalization were similar between arms, the native arm presented a antibodies high-titer associated with low asparaginase activity. As a result of this study, ${ }^{48}$ which demonstrated similar sustained depletion of serum ASN concentrations in patients receiving $\mathrm{PEG}-\mathrm{ASN}$ ase compared to those receiving EC L-ASNase, on July 24, 2006, the US Food and Drug Administration granted approval for PEG-ASNase for the first-line treatment of children with acute ALL as a component of a multiagent chemotherapy regimen. ${ }^{60}$

In addition to these pediatric studies on safety, tolerability and efficacy of PEG-ASNase in first-line treatment of ALL, studies focusing the administration schedule have been reported.

Silverman et al compared the use of EC L-ASNase 25,000 $\mathrm{IU} / \mathrm{m}^{2}$ weekly $3 \times 30$ doses and PEG-ASNase $2500 \mathrm{IU} / \mathrm{m}^{2}$ every 2 weeks $\times 15$ doses during intensification therapy. EFS at 5 years was $80 \%, 84 \% \pm 4 \%$ for native L-ASNase and $78 \% \pm 4 \%$ for PEG-ASNase-treated patients $(\mathrm{p}=0.29) .{ }^{92}$ The 5-year EFS for patients treated with less than 25 weeks of any L-ASNase vs at least 26 weeks of L-ASNase were $73 \% \pm 7 \%$ and $90 \% \pm 2 \%$, respectively $(\mathrm{p}=0.01)$.

The problem of the weekly or biweekly administration of PEG-ASNase has been studied also by Abshire who carried out a randomized phase II $^{95}$ comparing weekly with biweekly PEG-ASNase administration (2500 IU/m²) during ALL reinduction. In the chemotherapy schedule were also included vincristine $\left(1.5 \mathrm{mg} / \mathrm{m}^{2}\right.$ weekly $\times 4$ doses), doxorubicin (60 $\mathrm{mg} / \mathrm{m}^{2}$ on first day), prednisone $\left(40 \mathrm{mg} / \mathrm{m}^{2}\right.$ daily $\times 29$ days) and intrathecal medication (biweekly $\times 3$ doses from day 1 ). There was a highly significant difference $(\mathrm{p}=0.003)$ in second remission rates, with $97 \%$ (69 of 71) achieving complete response in the weekly group and $82 \%$ (60 of 73 ) in the every 2 weeks group.

Other pharmacokinetic and pharmacodynamic studies on PEG-ASNase were carried out in the pediatric population. ${ }^{16,96}$ The Italian group investigated the pharmacological effects of the administration of PEG-ASNase given as a first-line product in children with ALL. ${ }^{16}$ They investigated ASP serum enzymatic activity and serum and CSF levels of ASN in 20 children with newly diagnosed ALL treated with PEG-ASNase as a first line. The drug was administered during induction at the dosage of $1000 \mathrm{IU} / \mathrm{m}^{2}$ iv on days 12 and 27 and during reinduction only once at the same dosage. Among the 20 patients treated in induction serum PEG-ASNase activity was $\geq 100$ IU/L in $18 / 18,11 / 11$ and $15 / 18$ of the samples available on days 22, 25 and 27, respectively, and in 16/16, 12/15 and 5/8 samples available on days 36,39 and 45 , respectively. In the 15 patients treated during reinduction, serum PEG-ASNase activity $\geq 100 \mathrm{IU} / \mathrm{L}$ was observed in $14 / 15,11 / 14,6 / 10$, and $0 / 12$ samples available on days $11,15,18$ and 23 , respectively, after the administration of the drug. CSF asparagine levels were below the detection limit of the method only in a few patients during both induction and reinduction. They concluded that PEG-ASNase given as a first-line L-ASNase product allowed adequate plasma enzymatic activity and asparagine depletion during both exposures to the drug even if CSF asparagine depletion was inadequate.

These experiences with pediatric patients demonstrate that PEG-ASNase is safe and tolerable, with lower or similar frequency than other ASNase formulations of toxic reaction to EC L-ASNase for front-line treatment of children affected by ALL; it also has a more convenient administration schedule because of its prolonged half-life. There is no doubt that PEG-ASNase has improved treatment options for pediatric patients affected by ALL, but many questions remains especially regarding adult patients. As noted, the efficacy and toxicities of PEG-ASNase and EC L-ASNase in adult ALL have been compared only in nonrandomized trials. ${ }^{53,62,90,93}$ An important trial on feasibility, tolerability and efficacy of IV PEG-ASNase in adult ALL was carried out by Douer et $\mathrm{al}^{53}$ They studied pharmacodynamics and safety of IV PEG-ASNase during remission induction in 25 adult ALL administrating a single dose of $2000 \mathrm{IU} / \mathrm{m}^{2}$ as part of a standard frontline induction regimen. PEG-ASNase administered iv was well tolerated with no allergic reaction or pancreatitis and a long duration of ASN depletion. The largest nonrandomized clinical trial to date investigating the beneficial effect of PEG-ASNase in adult ALL was conducted by Wetzler et $\mathrm{al}^{62}$ The aim of the study was to explore differences in overall survival and disease-free survival of those patients who achieved ASN depletion compared with those who did not. They concluded that effective ASN depletion with PEG-ASNase as part of an intensive multiagent therapeutic regimen in ALL is feasible in adults and is associated with improved outcomes. These two studies confirm the feasibility and the efficacy of PEG-ASNase in first-line therapy also 
for adult ALL, but some questions remain. As concluded by Douer, the role of dose intensification especially in patients with anti L-ASNase antibodies, and prolonged duration of PEG-ASNase administration, need further study. Similarly, further studies are needed also to clarify the effects of intensifying the dose of PEG-ASNase to achieve prolonged CSF asparagines depletion.

A question that remains to be addressed is how anti L-ASNase and PEG antibodies formation affects ALL patient outcome. The effect of neutralizing antibodies on ALL outcome is controversial. The experience of Woo et al suggests there is no effect on the outcome of overall treatment in patients with hypersensitivity to the drug, ${ }^{98}$ but Panosyan et al showed that patients with neutralizing antibodies without signs of clinical allergy had a significantly worse outcome than patients who did not develop antibodies. ${ }^{56} \mathrm{Fu}$ et al suggest that alternating the various asparaginase formulations (PEG-ASNase, high dose EC L-ASNase and Erwinia ASNase) during the various phases of ALL therapy may help circumvent the production of neutralizing anti-L-ASNase antibodies. ${ }^{32}$ The problem of developing anti-PEG antibodies has to be considered as a potential risk of using a PEG-conjugated drug as a frontline treatment. As said before, Armstrong et al studied how antibodies against PEG adversely affect PEG-ASNase therapy in ALL patients. ${ }^{63}$ They tried to determine whether anti-PEG was associated with rapid clearance of PEG-ASNase in pediatric patients enrolled in the ALL Berlin-Frankfurt-Muenster 2000 studies. They concluded that the presence of anti-PEG was very closely associated with rapid clearance of $\mathrm{PEG}-\mathrm{ASN}$ ase, which explains similar observations with other PEG-conjugated drugs. ${ }^{99}$ For this reason they recommend that patients should be screened for pre-existing anti-PEG and routinely monitored for the development of anti-PEG in order to find patients for whom a modified dosing strategy or use of a nonPEGylated drug would be appropriate. The role of hypersensitivity and antibody development in the efficacy of PEG-ASNase in ALL treatment needs to be further explored in prospective clinical trials.

\section{Conclusions}

Many studies have demonstrated that the conjugation of L-ASNase with PEG has achieved the purpose of providing a long-duration form of the drug with a low grade of hypersensitivity. The advent of PEG-ASNase partially overcame the large limitation of immunogenic complications associated with native versions of the drug. This agent is very effective and for most patients needs to be administered only once every 2 weeks, as opposed to the 2 or 3 times a week for EC L-ASNase. Clearly, administering only 1 or 2 doses of a drug rather than 6 to 9 with the same anti-leukemic efficacy is considerably more convenient. Moreover the safe administration to patients with hypersensitivity to the $E$. coli drug makes it valuable for reinduction therapy in selected patients with ALL. The optimal usage of PEG-ASNase for the first-line treatment of patients affected by ALL remains to be determined precisely, especially considering the problem of dose intensification, drug resistance and development of anti PEG-ASNase antibodies. The monetary cost to patients of PEG-ASNase is greater than that of native versions, but the reduced complications of therapy make overall treatment cost considerably less than that of conventional preparations. ${ }^{100,101}$

In the near future it is hoped that the details for an individualized approach to therapy will be developed in order to exploit the maximum potential of this new important drug.

\section{Disclosures}

The authors report no conflicts of interest.

\section{References}

1. Apostolidou E, Swords R, Alvarado Y, et al. Treatment of acute lymphoblastic leukaemia: a new era. Drugs. 2007;67(15):2153-2171.

2. Pui CH, Evans WE. Treatment of acute lymphoblastic leukemia. NEngl J Med. 2006;354(2):166-178.

3. Gökbuget N, Hoelzer D. Treatment of adult acute lymphoblastic leukemia. Semin Hematol. 2009;46(1):64-75.

4. Ortega JA, Nesbit Jr ME, Donaldson MH, et al. 1-Asparaginase, vincristine and prednisone for induction of first remission in acute lymphoblastic leukemia. Cancer Res. 1977;37(2):535-540.

5. Jones B, Holland JF, Glidewell O, et al. Optimal use of 1-asparaginase (NSC-109229) in acute lymphoblastic leukemia. Med Pediatr Oncol. 1977;3(4):387-400.

6. Clavell LA, Gelber RD, Cohen HJ, et al. Four-agent induction and intensive 1-asparaginase therapy for treatment of childhood acute lymphoblastic leukemia. $N$ Engl J Med. 1986;315(11):657-663.

7. Schrappe M, Reiter A, Ludwig W, et al. Improved outcome in childhood acute lymphoblastic leukemia despite reduced use of anthracyclines cranial radiotherapy: results of trial ALL-BFM 90. Blood. 2000;95(11):3310-3322.

8. Hann I, Vora A, Richards S, et al. Benefit of intensified treatment for all children with acute lymphoblastic leukemia: results from MRC UKALL XI and MRC ALL97 randomized trials. Leukemia. 2000;14(3):356-363.

9. Kidd JG. Regression of transplanted lymphomas induced in vivo by means of normal guinea pig serum. I. Course of transplanted cancers of various kinds in mice and rats given guinea pig serum, horse serum, or rabbit serum. $J$ Exp Med. 1953;98(6):565-582.

10. Kidd JG. Regression of transplanted lymphomas induced in vivo by means of normal guinea pig serum. II. Studies on the nature of the active serum constituent: histological mechanism of the regression: tests for effects of guinea pig serum on lymphoma cells in vitro: discussion. $J$ Exp Med. 1953;98(6):583-606.

11. Mashburn LT, Wriston JC Jr. Tumor inhibitory effect of L-asparaginase from Escherichia coli. Arch Biochem Biophys. 1964;105:450-452. 
12. Pession A, Valsecchi MG, Masera G, et al. Long-term results of a randomized trial on extended use of high dose L-asparaginase for standard risk childhood acute lymphoblastic leukemia. J Clin Oncol. 2005;23(28):7161-7167.

13. Narta UK, Kanwar SS, Azmi W. Pharmacological and clinical evaluation of L-asparaginase in the treatment of leukemia. Crit Rev Oncol Hematol. 2007;61(3):208-221.

14. Ertel IJ, Nesbit ME, Hammond D, et al. Effective dose of L-asparaginase for induction of remission in previously treated children with acute lymphocytic leukemia: a report from Childrens Cancer Study Group. Cancer Res. 1979;39(10):3893-3896.

15. Nesbit M, Chard R, Evans A, et al. Evaluation of intramuscular versus intravenous administration of L-asparaginase in childhood leukemia. Am J Pediatr Hematol Oncol. 1979;1(1):9-13.

16. Rizzari C, Citterio M, Zucchetti M, et al. A pharmacological study on pegylated asparaginase used in front-line treatment of children with acute lymphoblastic leukemia. Haematologica. 2006;91(1):24-31.

17. Abuchowski A, Van Es T, Palczuk NC, et al. Treatment of L5178Y tumor-bearing BDF1 mice with a nonimmunogenic L-glutaminase-Lasparaginase. Cancer Treat Rep. 1979;63(6):1127-1132.

18. Yoshimoto T, Nishimura H, Saito Y, et al. Characterization of polyethylene glycol-modified L-asparaginase from Escherichia coli and its application to therapy of leukemia. Jpn J Cancer Res. 1986;77(12):1264-1270.

19. Veronese FM, Mero A. The impact of PEGylation on biological therapies. Bio Drugs. 2008;22(5):315-329.

20. Duval M, Suciu S, Ferster A, et al. Comparison of Escherichia coli-asparaginase with Erwinia-asparaginase in the treatment of childhood lymphoid malignancies: results of a randomized European Organisation for Research and Treatment of Cancer-Children's Leukemia Group phase 3 trial. Blood. 2002;99(8):2734-2739.

21. Campbell HA, Mashburn LT, Boyse EA, et al. Two L-asparaginases from Escherichia coli B. Their separation, purification, and antitumor activity. Biochemistry. 1967;6(3):721-730.

22. Jackson RC, Handschumacher RE. Escherichia coli L-asparaginase. Catalytic activity and subunit nature. Biochemistry. 1970;9(18):3585-3590.

23. Maita T, Matsuda G. The primary structure of L-asparaginase from Escherichia coli. Hoppe Seylers Z Physiol Chem. 1980;361(2):105-117.

24. Whelan HA, Wriston JC Jr. Purification and properties of asparaginase from escherichia coli B. Biochemistry. 1969;8(6):2386-2393.

25. Chabner BA, Loo TL. Enzyme therapy: L-asparaginase. In: Cancer Chemotherapy and Biotherapy Principles. Chabner BA, Longo DL, Editors. Philadelphia: Lippincot-Raven; 1966. p. 485-492.

26. Swain AL, Jaskólski M, Housset D, et al. Crystal structure of Escherichia coli L-asparaginase, an enzyme used in cancer therapy. Proc Natl Acad Sci US A. 1993;90(4):1474-1478.

27. Molineux G. Pegylation: engineering improved pharmaceuticals for enhanced therapy. Cancer Treat Rev. 2002;28 Suppl A:13-16.

28. Abuchowski A, Kazo GM, Verhoest CR Jr, et al. Cancer therapy with chemically modified enzymes. I. Antitumor properties of polyethylene glycol-asparaginase conjugates. Cancer Biochem Biophys. 1984;7(2):175-186.

29. Kamisaki Y, Wada H, Yagura T, et al. Reduction in immunogenicity and clearance rate of Escherichia coli L-asparaginase by modification with monomethoxypolyethylene glycol. J Pharmacol Exp Ther. 1981;216(2):410-414

30. Wada H, Imamura I, Sako M, et al. Antitumor enzyme: polyethylene glycol-modified asparaginase. Ann N Y Acad Sci. 1990;613:95-108.

31. Keating MJ, Holmes R, Lerner S, et al. L-asparaginase and PEG asparaginase - past, present, and future. Leuk Lymphoma. 1993;10 Suppl:153-157.

32. $\mathrm{Fu} \mathrm{CH}$, Sakamoto KM. PEG-ASNase araginase. Expert Opin Pharmacother. 2007;8(12):1977-1984.

33. Ettinger LJ, Kurtzberg J, Voûte PA, et al. An open-label, multicenter study of polyethylene glycol-L-asparaginase for the treatment of acute lymphoblastic leukemia. Cancer. 1995;75(5):1176-1181.
34. Vieira Pinheiro JP, Wenner K, Escherich G, et al. Serum asparaginase activities and asparagine concentrations in the cerebrospinal fluid after a single infusion of 2,500 IU/m(2) PEG asparaginase in children with ALL treated according to protocol COALL-06-97. Pediatr Blood Cancer. 2006;46(1):18-25.

35. Hak LJ, Relling MV, Cheng C, et al. Asparaginase pharmacodynamics differ by formulation among children with newly diagnosed acute lymphoblastic leukemia. Leukemia. 2004;18(6):1072-1077.

36. Müller HJ, Beier R, da Palma JC, et al. PEG-ASNase araginase (Oncaspar) $2500 \mathrm{U} / \mathrm{m}(2) \mathrm{BSA}$ in reinduction and relapse treatment in the ALL/NHL-BFM protocols. Cancer Chemother Pharmacol. 2002;49(2):149-154

37. Kiriyama Y, Kubota M, Takimoto T, et al. Biochemical characterization of U937 cells resistant to L-asparaginase: the role of asparagine synthetase. Leukemia. 1989;3(4):294-297.

38. Prager MD, Bachynsky N. Asparagine synthetase in asparaginase resistant and susceptible mouse lymphomas. Arch Biochem Biophys. 1968;127(1):645-654.

39. Haskell CM, Canellos GP, Cooney DA, et al. Biocmical and pharmacologic effects of L-asparaginase in man. J Lab Clin Med. 1970;75(5):763-770.

40. Cooney DA, Handschumacher RE. L-asparaginase and L-asparagine metabolism. Annu Rev Pharmacol. 1970;10:421-440.

41. Capizzi RL, Bertino JR, Skeel RT, et al. L-asparaginase: clinical, biochemical, pharmacological, and immunological studies. Ann Intern Med. 1971;74(6):893-901.

42. Asselin BL, Ryan D, Frantz CN, et al. In vitro and in vivo killing of acute lymphoblastic leukemia cells by L-asparaginase. Cancer Res. 1989;49(15):4363-4368.

43. Ueno T, Ohtawa K, Mitsui K, et al. Cell cycle arrest and apoptosis of leukemia cells induced by L-asparaginase. Leukemia. 1997;11(11):1858-18561.

44. Shimizu T, Kubota M, Adachi S, et al. Pre-treatment of a human T-lymphoblastoid cell line with L-asparaginase reduces etoposide-induced DNA strand breakage and cytotoxicity. Int $J$ Cancer. 1992;50(4):644-648.

45. Capizzi RL, Aggarwal K. Asparaginase. In Biochemical Pharmacology of Blood and Blood Forming Agents. Fisher JW, editor. Heidelberg: Springer; 1992. p. 548-565.

46. Park YK, Abuchowski A, Davis S, et al. Pharmacology of Escherichia coli-L-asparaginase polyethylene glycol adduct. Anticancer Res. 1981;1(6):373-376.

47. Berg SL, Balis FM, McCully CL, et al. Pharmacokinetics of PEG-L-asparaginase and plasma and cerebrospinal fluid L-asparagine concentrations in the rhesus monkey. Cancer Chemother Pharmacol. 1993;32(4):310-314.

48. Avramis VI, Sencer S, Periclou AP, et al. A randomized comparison of native Escherichia coli asparaginase and polyethylene glycol conjugated asparaginase for treatment of children with newly diagnosed standardrisk acute lymphoblastic leukemia: a Children's Cancer Group study. Blood. 2002;99(6):1986-94. Erratum in Blood. 2002;100(5):1531.

49. Ho DH, Brown NS, Yen A, et al. Clinical pharmacology of polyethylene glycol-L-asparaginase. Drug Metab Dispos. 1986;14(3):349-352.

50. Asselin BL, Whitin JC, Coppola DJ, et al. Comparative pharmacokinetic studies of three asparaginase preparations. Clin Oncol. 1993;11(9):1780-1786.

51. Holle LM. Pegaspargase: an alternative? Ann Pharmacother. 1997;31(5): 616-624.

52. Albertsen BK, Jakobsen P, Schrøder H, et al. Pharmacokinetics of Erwinia asparaginase after intravenous and intramuscular administration. Cancer Chemother Pharmacol. 2001;48(1):77-82.

53. Douer D, Yampolsky H, Cohen LJ, et al. Pharmacodynamics and safety of intravenous pegaspargase during remission induction in adults aged 55 years or younger with newly diagnosed acute lymphoblastic leukemia. Blood. 2007;109(7):2744-2750.

54. Wenner KA, Vieira Pinheiro JP, Escherich G, et al. Asparagine concentration in plasma after 2,500 IU/ $/ \mathrm{m}^{2}$ PEG-ASNase araginase i.v. in children with acute lymphoblastic leukemia. Klin Padiatr. 2005;217(6):321-326. 
55. Capizzi RL. Asparaginase revisited. Leuk Lymphoma. 1993; 10 Suppl:147-150.

56. Panosyan EH, Seibel NL, Martin-Aragon S, et al. Asparaginase antibody and asparaginase activity in children with higher-risk acute lymphoblastic leukemia: Children's Cancer Group Study CCG-1961. J Pediatr Hematol Oncol. 2004;26(4):217-226.

57. Ohnuma T, Holland JF, Freeman A, et al. Biochemical and pharmacological studies with asparaginase in man. Cancer Res. 1970;30(9):2297-2305.

58. Cheung NK, Chau IY, Coccia PF. Antibody response to Escherichia coli L-asparaginase. Prognostic significance and clinical utility of antibody measurement. Am J Pediatr Hematol Oncol. 1986;8(2):99-104.

59. Appel IM, Pinheiro JP, den Boer ML, et al. Lack of asparagine depletion in the cerebrospinal fluid after one intravenous dose of PEG-ASNase araginase: a window study at initial diagnosis of childhood ALL. Leukemia. 2003;17(11):2254-2256.

60. Dinndorf PA, Gootenberg J, Cohen MH, et al. FDA drug approval summary: pegaspargase (oncaspar) for the first-line treatment of children with acute lymphoblastic leukemia (ALL). Oncologist 2007;12(8):991-998.

61. Avramis VI, Panosyan EH. Pharmacokinetic/pharmacodynamic relationships of asparaginase formulations: the past, the present and recommendations for the future. Clin Pharmacokinet. 2005;44(4):367-393.

62. Wetzler M, Sanford BL, Kurtzberg J, et al. Effective asparagine depletion with pegylated asparaginase results in improved outcomes in adult acute lymphoblastic leukemia: Cancer and Leukemia Group B Study 9511. Blood. 2007;109(10):4164-4167.

63. Armstrong JK, Hempel G, Koling S, et al. Antibody against poly(ethylene glycol) adversely affects PEG-ASNase araginase therapy in acute lymphoblastic leukemia patients. Cancer. 2007;110(1):103-111.

64. Hak LJ, Relling MV, Cheng C, et al. Asparaginase pharmacodynamics differ by formulation among children with newly diagnosed acute lymphoblastic leukemia. Leukemia. 2004;18(6):1072-1077.

65. Haskell CM, Canellos GP. 1-asparaginase resistance in human leukemiaasparagine synthetase. Biochem Pharmacol. 1969;18(10):2578-2580.

66. Broome JD, Schwartz JH. Differences in the production of L-asparagine in asparaginase-sensitive and resistant lymphoma cells. Biochim Biophys Acta. 1967;138(3):637-639.

67. Prager MD, Bachynsky N. Asparagine synthetase in asparaginase resistant and susceptible mouse lymphomas. Biochem Biophys Res Commun. 1968;31(1):43-47.

68. Kiriyama Y, Kubota M, Takimoto T, et al. Biochemical characterization of U937 cells resistant to L-asparaginase: the role of asparagine synthetase. Leukemia. 1989;3(4):294-297.

69. Hutson RG, Kitoh T, Moraga Amador DA, et al. Amino acid control of asparagine synthetase: relation to asparaginase resistance in human leukemia cells. Am J Physiol. 1997;272(5 Pt 1):C1691-C1699.

70. Gallagher MP, Marshall RD, Wilson R. Asparaginase as a drug for treatment of acute lymphoblastic leukaemia. Essays Biochem. 1989;24:1-40.

71. Holleman A, den Boer ML, Kazemier KM, et al. Resistance to different classes of drugs is associated with impaired apoptosis in childhood acute lymphoblastic leukemia. Blood. 2003;102(13):4541-4546.

72. Faderl S, Thall PF, Kantarjian HM, et al. Caspase 2 and caspase 3 as predictors of complete remission and survival in adults with acute lymphoblastic leukemia. Clin Cancer Res. 1999;5(12):4041-4047.

73. Gallagher MP, Marshall RD, Wilson R. 1-Asparaginase a drug for treatment of acute lymphoblastic leukemia. Essays Biochem. 1989; 24:1-40.

74. Haskell CM, Canellos GP, Leventhal BG, et al. L-asparaginase: therapeutic and toxic effects in patients with neoplastic disease. $N$ Engl $J$ Med. 1969;281(19):1028-1034.

75. Package Insert. Oncaspar (pegaspargase), Rhone-Pooulenc Rorer, Collegeville, PA; 1994.

76. Oettgen HF, Tallal L, Tan CC, et al. Experimental and clinical effects of 1-asparaginase. In: Grundman E, Oettegen HF, editors. Clinical Experience with L-asparaginase. New York: Springer; 1970. p. 219-43.
77. Anderson N, Lokich JJ, Tullis JL. L-asparaginase effect on antithrombin-III levels. Med Pediatr Oncol. 1979;7(4):335-340.

78. Priest JR, Ramsay NKC, Bennet AJ, et al. The effect of 1-asparaginase on antithrombin, plasminogen and plasma coagulation during therapy for acute lymphoblastic leukemia. J Pediatr. 1982;100(6): 990-995.

79. Homans AC, Rybak ME, Baglini RL, et al. Effect of 1-asparaginase administration on coagulation and platelet function in children with leukemia. J Clin Oncol. 1987;5(5):811-817.

80. Gugliotta L, D'Angelo A, Mattioli-Belmonte M, et al. Hypercoagulability during 1-asparaginase treatment: the effect of antithrombin III supplementation in vivio. Br J Hematol. 1990;74(4):465-470.

81. Wermes C, von Depka Prondzinski M, Lichtinghagen R, et al. Clinical relevance of genetic risk factors for thrombosis in paediatric oncology patients with central venous catheters. Eur J Pediatr. 1999;158 Suppl 3: S143-S146.

82. Knofler R, Siegert E, Lauterbach I, et al. Clinical importance of prothrombotic risk factors in pediatric patients with malignancy-impact of central venous lines. Eur J Pediatr. 1999;158 Suppl 3:S147-S150.

83. Mauz-Körholz C, Junker R, Göbel U, et al. Prothrombotic risk factors in children with acute lymphoblastic leukemia treated with delayed $\mathrm{E}$. coli asparaginase (COALL-92 and 97 protocols). Thromb Haemost. 2000;83(6):840-843.

84. Nguyen DL, Wilson DA, Engleman ED, et al. Serial sonogram to detect pancreatitis in children receiving 1-asparaginase. South Med J. 1987;80(9):1133-1136.

85. Samuels BI, Culbert SJ, Okamura J, et al. Early detection of chemotherapy-related pancreatic enlargement in children using abdominal sonography: a preliminary report. Cancer. 1976;38(4):1515-1523.

86. Pochedly C. Neurotoxicity due to CNS therapy for leukemia. Med Pediatr Oncol. 1977;3(1):101-115.

87. Vieira Pinheiro JP, Müller HJ, Schwabe D, et al. Drug monitoring of low-dose PEG-ASNase araginase (Oncaspar) in children with relapsed acute lymphoblastic leukaemia. Br J Haematol. 2001;113(1): $115-119$.

88. Taylor CW, Dorr RT, Fanta P, et al. A phase I and pharmacodynamic evaluation of polyethylene glycol-conjugated L-asparaginase in patients with advanced solid tumors. Cancer Chemother Pharmacol. 2001;47(1):83-88.

89. Aguayo A, Cortes J, Thomas D, et al. Combination therapy with methotrexate, vincristine, polyethylene-glycol conjugated-asparaginase, and prednisone in the treatment of patients with refractory or recurrent acute lymphoblastic leukemia. Cancer. 1999;86(7):1203-1209.

90. Douer D, Cohen LJ, Peridou LA, et al. Peg-L-asparaginase (PEGASNASE) : Pharmacokinetics (PK) and clinical response in newly diagnosed adults with acute lymphoblastic leukemia (ALL) treated with multiagent chemotherapy Blood. 1997;90(Suppl 1):334a.

91. Muss HB, Spell N, Scudiery D, et al. A phase II trial of PEG-Lasparaginase in the treatment of non-Hodgkins lymphoma. Invest New Drugs. 1990;8(1):125-130.

92. Silverman LB, Gelber RD, Dalton VK, et al. Improved outcome for children with acute lymphoblastic leukemia: results of Dana-Farber Consortium Protocol 91-01. Blood. 2001;97(5):1211-1218.

93. Rosen O, Müller HJ, Gökbuget N, et al. Pegylated asparaginase in combination with high-dose methotrexate for consolidation in adult acute lymphoblastic leukaemia in first remission: a pilot study. $\mathrm{Br} \mathrm{J}$ Haematol. 2003;123(5):836-841.

94. Holcenberg J, Sencer S, Cohen LJ, et al. Randomised trial of PEG vs native 1-asparaginase in children with newly diagnosed acute lymphoblastic leukemia (ALL): CCG study 1962. Blood. 1999;94:628a.

95. Abshire TC, Pollock BH, Billett AL, et al. Weekly polyethylene glycol conjugated L-asparaginase compared with biweekly dosing produces superior induction remission rates in childhood relapsed acute lymphoblastic leukemia: a Pediatric Oncology Group Study. Blood. 2000;96(5):1709-1715. 
96. Appel IM, Kazemier KM, Boos J, et al. Pharmacokinetic, pharmacodynamic and intracellular effects of PEG-ASNase araginase in newly diagnosed childhood acute lymphoblastic leukemia: results from a single agent window study. Leukemia. 2008;22(9):1665-1679.

97. Zeidan A, Wang ES, Wetzler M. Pegasparaginase: where do we stand? Expert Opin Biol Ther. 2009;9(1):111-119.

98. Woo MH, Hak LJ, Storm MC, et al. Hypersensitivity or development of antibodies to asparaginase does not impact treatment outcome of childhood acute lymphoblastic leukemia. J Clin Oncol. 2000;18(7):1525-1532.

99. van der Eijk AA, Vrolijk JM, Haagmans BL. Antibodies neutralizing peginterferon alfa during retreatment of hepatitis C. $N$ Engl J Med. 2006;354(12):1323-1324.
100. Peters BG, Goeckner BJ, Ponzillo JJ, et al. Pegaspargase versus asparaginase in adult ALL: a pharmacoeconomic assessment. Formulary. 1995;30(7):388-393.

101. Kurre HA, Ettinger AG, Veenstra DL, et al. A pharmacoeconomic analysis of pegaspargase versus native Escherichia coli L-asparaginase for the treatment of children with standard-risk, acute lymphoblastic leukemia: the Children's Cancer Group study (CCG-1962). J Pediatr Hematol Oncol. 2002;24(3):175-181.

\section{Publish your work in this journal}

Biologics: Targets \& Therapy is an international, peer-reviewed journal focusing on the patho-physiological rationale for and clinical application of Biologic agents in the management of autoimmune diseases, cancers or other pathologies where a molecular target can be identified. This journal is indexed on PubMed Central, CAS,

\section{Dovepress}

EMBase, Scopus and the Elsevier Bibliographic databases. The manuscript management system is completely online and includes a very quick and fair peer-review system, which is all easy to use. Visit http://www.dovepress.com/testimonials.php to read real quotes from published authors 